\title{
Modelo Estocástico do Algoritmo LMS de Passo Variável Baseado na Autocorrelação do Erro com $N$ Lags
}

\author{
José Gil Fausto Zipf, Orlando José Tobias e Rui Seara
}

\begin{abstract}
Resumo-Este trabalho propõe um modelo estocástico do comportamento médio do vetor de coeficientes e da curva de aprendizagem para o algoritmo LMS de passo variável baseado na autocorrelação do sinal de erro usando $N$ lags. Esse algoritmo consiste em um aprimoramento do algoritmo proposto por Aboulnasr, visando ambientes com baixa razão sinal-ruído (SNR). Uma característica importante do algoritmo usando autocorrelação de $N$ lags é o seu bom desempenho em tais ambientes às custas de um pequeno acréscimo de complexidade computacional. Simulações numéricas confirmam a boa precisão do modelo desenvolvido. Adicionalmente, é também discutida uma proposta de redução da carga computacional do algoritmo em questão.
\end{abstract}

Palavras-chave-Algoritmo LMS de passo variável, comportamento médio do vetor de coeficientes, curva de aprendizagem, filtros adaptativos, modelo estocástico.

Abstract-This paper proposes a stochastic model of the mean weight behavior and the learning curve for the variable step-size LMS (VSSLMS) algorithm based on the error signal autocorrelation using $N$ lags. This algorithm consists of an enhancement of the Aboulnasr's algorithm, aiming at environment with low signal-to-noise ratio (SNR). An important characteristic of the algorithm using autocorrelation of $N$ lags is its very good performance for such environment at the expense of a small increase in the computational complexity. Numerical simulations confirm the good accuracy of proposed stochastic model. In addition, a scheme for computational burden reduction of this algorithm is discussed.

Keywords-Variable step-size LMS algorithm, mean weight behavior, learning curve, adaptive filters, stochastic model.

\section{INTRODUÇÃO}

A grande popularidade do algoritmo LMS se deve principalmente à sua reduzida complexidade computacional e muito boa estabilidade [1]. Tais vantagens tornam o algoritmo LMS adequado para diversas aplicações em filtragem adaptativa, tais como identificação de sistemas, cancelamento de ruído, cancelamento de eco, equalização de

José Gil F. Zipf, Orlando J. Tobias e Rui Seara, LINSE - Laboratório de Circuitos e Processamento de Sinais, Depto. de Eng. Elétrica, Universidade Federal de Santa Catarina, Florianópolis, SC, E-mails: \{gil, orlando, seara\}@linse.ufsc.br.

José Gil F. Zipf e Orlando J. Tobias são também Professores do Departamento de Engenharia Elétrica e Telecomunicações da Universidade Regional de Blumenau (FURB), Blumenau, SC, Brasil.

Este trabalho foi parcialmente financiado pelo Conselho Nacional de Desenvolvimento Científico e Tecnológico $(\mathrm{CNPq})$. canal, dentre outras [2]. No algoritmo LMS convencional, existe um compromisso na escolha do passo de adaptação, visto que o valor do passo afeta de forma decisiva o desempenho do algoritmo. Assim, um maior valor de passo leva a uma maior velocidade de convergência às custas de um maior desajuste do algoritmo. Por outro lado, um valor pequeno de passo implica um menor desajuste do algoritmo, levando a uma taxa de convergência mais baixa. Como conseqüência, diferentes algoritmos LMS de passo variável vêm sendo propostos com o objetivo de mitigar o compromisso inerente à seleção do passo de adaptação. Tais algoritmos visam a obtenção de uma alta velocidade de convergência aliada a um reduzido desajuste, sendo denominados na literatura algoritmos VSSLMS (variable step-size LMS). A idéia central desse tipo de algoritmo é utilizar maiores valores de passo no início da convergência e reduzi-lo gradualmente (segundo algum dado critério) à medida que o regime permanente se aproxime. Diferentes estratégias de ajuste do passo de adaptação para algoritmos VSSLMS vêm sendo propostas, as quais são baseadas nos mais diversos princípios, a saber: gradiente do erro quadrático [3]-[9], erro quadrático instantâneo [10]-[12], função de autocorrelação do erro [13], [14], erro de adaptação absoluto [15], normalização do vetor de erro [16], valor absoluto do vetor de coeficientes [17]-[19], e outros [20]. Em [13], é proposto um algoritmo baseado na medida de autocorrelação do sinal de erro $\operatorname{lag}(1)$, apresentando uma muito boa imunidade ao ruído não-correlacionado de medição. Tal algoritmo é aqui denominado algoritmo de Aboulnasr. Em [14], uma versão melhorada desse algoritmo, em termos de imunidade ao ruído de medição, é proposta. A idéia básica desse algoritmo é considerar autocorrelação do sinal de erro calculada para vários $\operatorname{lags}, \operatorname{lag}(1), \operatorname{lag}(2), \ldots, \operatorname{lag}(N), \operatorname{com}$ $N-1$ denotando a ordem do filtro adaptativo. Esse algoritmo consiste em um aprimoramento do algoritmo proposto por Aboulnasr, visando ambientes com baixa razão sinal-ruído (SNR). Uma relevante característica do algoritmo usando autocorrelação de $N$ lags é o seu bom desempenho em tais ambientes às custas de um pequeno acréscimo de carga computacional [14].

Este trabalho apresenta um modelo estocástico do comportamento médio do vetor de coeficientes e da curva de aprendizagem para o algoritmo LMS de passo variável apresentado em [14]. Simulações numéricas corroboram a 
boa precisão do modelo desenvolvido. Além do modelo apresentado, é também discutida uma proposta de redução da carga computacional do algoritmo em questão.

\section{Algoritmos VSSLMS}

O uso de uma estratégia de passo de adaptação variável (variable step-size - VSS) melhora consideravelmente o desempenho do algoritmo LMS convencional, possibilitando a obtenção simultânea de maior taxa de convergência e um reduzido desajuste.

Considere então um problema de identificação de sistema, ilustrado pelo diagrama de blocos da Fig. 1 .

O sinal de saída do sistema desconhecido, corrompido por um ruído de medição, é dado por

$$
d(n)=\mathbf{w}_{\mathrm{o}}^{\mathrm{T}} \mathbf{x}(n)+\eta(n)
$$

onde $\mathbf{x}(n)=[x(n) x(n-1) \cdots x(n-N+1)]^{\mathrm{T}}$ denota o vetor de entrada, sendo $\{x(n)\}$ um processo gaussiano de média zero e variância $\sigma_{x}^{2}, \eta(n)$ é um ruído de medição i.i.d com variância $\sigma_{\eta}^{2}, \mathbf{w}(n)=\left[w_{0}(n) w_{1}(n) \cdots w_{N-1}(n)\right]^{\mathrm{T}}$ caracteriza o vetor de coeficientes e $\mathbf{w}_{\mathrm{o}}=\left[\begin{array}{llll}w_{0} & w_{1} & \cdots & w_{N-1}\end{array}\right]^{\mathrm{T}}$, a planta do sistema a ser identificado. $O$ sinal de erro é dado por

$$
e(n)=d(n)-\mathbf{w}^{\mathrm{T}}(n) \mathbf{x}(n) .
$$

A expressão de atualização do vetor de coeficientes para algoritmos VSSLMS é escrita como

$$
\mathbf{w}(n+1)=\mathbf{w}(n)+\mu(n) e(n) \mathbf{x}(n)
$$

onde $\mu(n)$ é o passo de adaptação variável. A diferença entre os diversos algoritmos VSSLMS está na forma de obtenção do passo de adaptação variável. Para garantir uma operação estável do algoritmo adaptativo, os limites de ajuste do passo de adaptação são fixados pela seguinte inequação [2]:

$$
0<\mu(n)<\frac{2}{3 \operatorname{tr}[\mathbf{R}]}
$$

onde $\mathbf{R}$ é a matriz de autocorrelação do vetor de entrada.

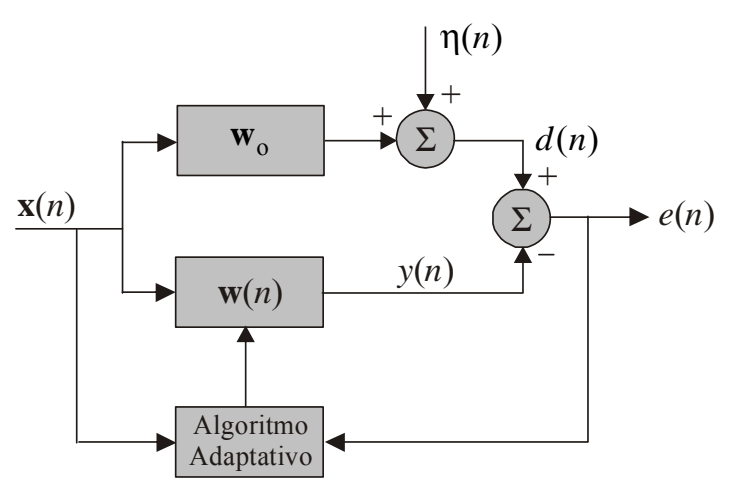

Fig. 1. Diagrama de blocos de um esquema de identificação de sistema.

\section{AlgOritmo DE ABOUlnASR}

Este algoritmo realiza o ajuste do passo de adaptação com base na medida de autocorrelação entre o sinal $e(n)$ e $e(n-1)$. Essa abordagem fornece uma razoável imunidade ao ruído aditivo não-correlacionado de medição se comparada à estratégia baseada no sinal de erro ao quadrado [10]. A atualização do passo de adaptação é baseada no quadrado da estimativa da autocorrelação do sinal de erro, obtida por

$$
p(n)=\beta p(n-1)+(1-\beta) e(n) e(n-1) .
$$

Essa operação representa uma filtragem passa-baixas, onde $\beta>0$ é um parâmetro de controle. Finalmente, a expressão de atualização do passo de adaptação variável é dada por

$$
\mu(n+1)=\alpha \mu(n)+\gamma p^{2}(n)
$$

onde $\alpha$ e $\gamma$ são parâmetros positivos de controle.

\section{Algoritmo VSSLMS BASEAdo NA AUtocorRelaÇÃo DO ERRO COM $N$ LAGS}

A utilização da função de autocorrelação entre $e(n)$ e $e(n-1)$, discutida em [13], pode (em algumas situações) não ser a melhor forma para efetuar o ajuste do passo de adaptação. Dependendo da correlação do sinal de entrada e do tipo de resposta ao impulso do sistema a ser identificado, a autocorrelação entre $e(n)$ e $e(n-2), e(n)$ e $e(n-3)$ e outros valores de lag pode ser tão ou mais significativa do que a autocorrelação do erro $\operatorname{lag}(1)$. Assim, no algoritmo de Aboulnasr [13], podem ocorrer situações em que o passo de adaptação é reduzido antecipadamente, deixando o algoritmo desnecessariamente mais lento do que o requerido.

O algoritmo VSSLMS baseado na autocorrelação do erro usando $N$ lags proposto em [14] usa $N$ medidas de autocorrelações do erro. Com isso, o processo de convergência é acelerado, mantendo uma maior imunidade ao ruído de medição.

Então, consideremos $p(n)$, valor médio da soma dos quadrados das funções de autocorrelação entre $e(n)$ e $e(n-1), e(n-2), \ldots, e(n-N)$, estimado através da seguinte expressão:

$$
p(n)=\beta p(n-1)+(1-\beta) \sum_{i=1}^{N}[e(n) e(n-i)]^{2}
$$

onde $\beta>0$ é um parâmetro de controle. A equação de adaptação do passo variável é agora dada por

$$
\mu(n+1)=\alpha \mu(n)+\gamma p(n)
$$

onde $\alpha$ e $\gamma$ são parâmetros usados para controlar o ajuste do passo de adaptação. A utilização de vários atrasos $[\operatorname{lag}(1)$, $\operatorname{lag}(2), \ldots, \operatorname{lag}(N)]$, considerado pelo algoritmo discutido em [14], leva a um aumento na complexidade computacional em comparação com aquela do algoritmo de Aboulnasr. A Tabela I apresenta a complexidade computacional de ambos os algoritmos, possibilitando uma análise comparativa. 
TABELA I

COMPARAÇÃO DA COMPLEXIDADE COMPUTACIONAL

\begin{tabular}{c|c|c}
\hline \hline Algoritmo & $\begin{array}{c}\text { Número de } \\
\text { Multiplicações }\end{array}$ & $\begin{array}{c}\text { Número de Somas } \\
\text { e Subtrações }\end{array}$ \\
\hline Aboulnasr & $2 N+7$ & $2 N+2$ \\
\hline Ref. [14] & $3 N+7$ & $3 N+1$ \\
\hline \hline
\end{tabular}

Uma estratégia alternativa de atualização do passo de adaptação variável pode ser então utilizada, levando a uma redução na carga computacional do algoritmo VSSLMS. Tal estratégia implica atualizar, a cada iteração, apenas uma das diversas medidas de autocorrelação do sinal de erro. Um resumo dessa estratégia é apresentado na Tabela II.

TABELA II

PRINCIPAIS PASSOS DO ALGORITMO VSSLMS [14]

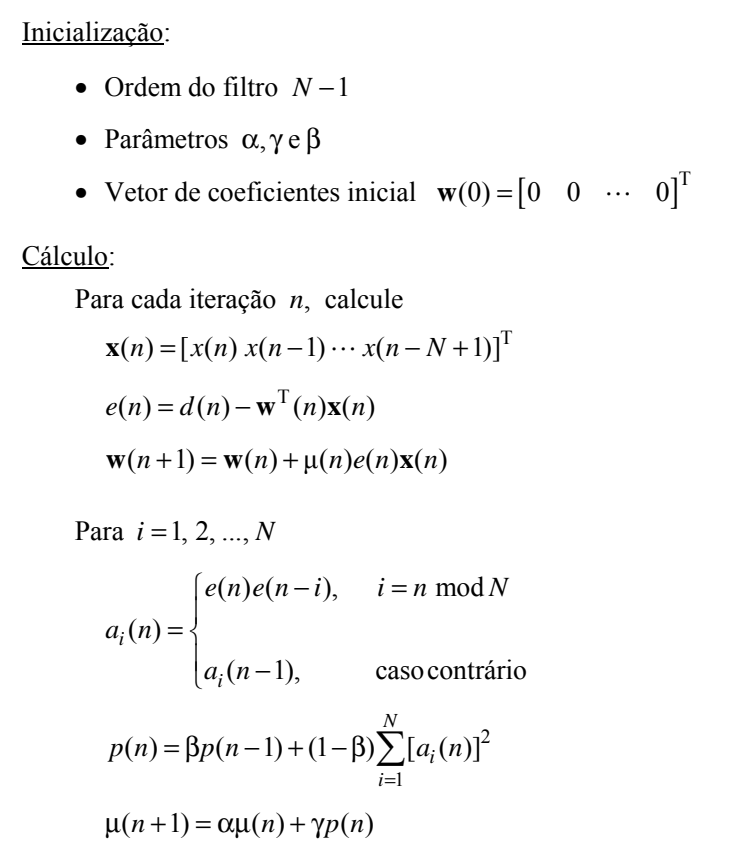

Com este procedimento, a complexidade computacional do algoritmo apresentado em [14] seria reduzida para $2 N+8$ operações de multiplicações e $2 N+3$ operações de somas.

\section{Modelagem}

Nesta seção, um modelo analítico para o algoritmo VSSLMS proposto em [14] é derivado. São obtidas expressões de predição do comportamento médio do vetor de coeficientes e da curva de aprendizagem do algoritmo analisado. Tais expressões são derivadas invocando parcialmente a Teoria da Independência (TI) e assumindo as seguintes hipóteses simplificativas:

i) O passo variável de adaptação $\mu(n)$ é independente e descorrelacionado de qualquer outro sinal no sistema.

ii) $E\left[\mu^{2}(n)\right]=E^{2}[\mu(n)]$.

iii) $E[\mu(n) \mu(n-i)]=E[\mu(n)] E[\mu(n-i)], \quad i=1 \ldots N$

iv) $E\left\{[e(n) e(n-i)]^{2}\right\}=E^{2}[e(n) e(n-i)], \quad i=1 \ldots N$.
As hipóteses anteriormente consideradas são válidas sob condições de adaptação lenta do passo $\mu(n)$.

\section{A. Comportamento Médio do Vetor de Coeficientes}

O modelo para o comportamento médio do vetor de coeficientes é obtido tomando o valor esperado de (3) e considerando (i). Então,

$$
E[\mathbf{w}(n+1)]=E[\mathbf{w}(n)]+E[\mu(n)]\{\mathbf{p}-\mathbf{R} E[\mathbf{w}(n)]\}
$$

onde $\mathbf{R}=E\left[\mathbf{x}(n) \mathbf{x}^{\mathrm{T}}(n)\right]$ é a matriz de autocorrelação do vetor de entrada e $\mathbf{p}=E[d(n) \mathbf{x}(n)]$, o vetor de correlação cruzada entre o sinal desejado e o vetor de entrada. A variável $E[\mu(n)]$ precisa, então, ser determinada em (9). Tomando o valor esperado de ambos os lados de (8), obtém-se

$$
E[\mu(n+1)]=\alpha E[\mu(n)]+\gamma E[p(n)] .
$$

Para calcular $E[p(n)]$ em (10), toma-se o valor esperado de ambos os lados de (7). Dessa forma,

$$
E[p(n)]=\beta E[p(n-1)]+(1-\beta) \sum_{i=1}^{N} E\left\{[e(n) e(n-i)]^{2}\right\} .
$$

Agora, considerando (iv), (11) pode ser reescrita como

$$
E[p(n)]=\beta E[p(n-1)]+(1-\beta) \sum_{i=1}^{N} E^{2}[e(n) e(n-i)]
$$

onde $\sum_{i=1}^{N} E^{2}[e(n) e(n-i)]$ precisa ser determinado em

Para tal, considere o vetor de erro nos coeficientes dado por

$$
\mathbf{v}(n)=\mathbf{w}(n)-\mathbf{w}_{\mathrm{o}} .
$$

Assim, os sinais de erro $e(n)$ e $e(n-i)$ podem ser escritos, respectivamente, como

$$
e(n)=\eta(n)-\mathbf{v}^{\mathrm{T}}(n) \mathbf{x}(n)
$$

e

$$
e(n-i)=\eta(n-i)-\mathbf{v}^{\mathrm{T}}(n-i) \mathbf{x}(n-i) .
$$

Agora, usando (14) e (15), a função de autocorrelação com $\operatorname{lag}(i)$ é obtida de

$$
\begin{aligned}
e(n) e(n-i)= & \eta(n) \eta(n-i)-\eta(n) \mathbf{v}^{\mathrm{T}}(n-i) \mathbf{x}(n-i) \\
& -\eta(n-i) \mathbf{v}^{\mathrm{T}}(n) \mathbf{x}(n) \\
& +\mathbf{v}^{\mathrm{T}}(n) \mathbf{x}(n) \mathbf{x}^{\mathrm{T}}(n-i) \mathbf{v}(n-i) .
\end{aligned}
$$

Então, tomando o valor esperado de ambos os lados de (16)

$$
E[e(n) e(n-i)]=E\left[\mathbf{v}^{\mathrm{T}}(n) \mathbf{x}(n) \mathbf{x}^{\mathrm{T}}(n-i) \mathbf{v}(n-i)\right]
$$

e definindo a matriz de correlação entre os vetores $\mathbf{x}(n)$ e $\mathbf{x}(n-i)$ como

$$
\mathbf{R}_{i}=E\left[\mathbf{x}(n) \mathbf{x}^{\mathrm{T}}(n-i)\right]
$$

(17) pode ser obtida por 


$$
E[e(n) e(n-i)]=E\left[\mathbf{v}^{\mathrm{T}}(n) \mathbf{R}_{i} \mathbf{v}(n-i)\right]
$$

Note que a matriz $\mathbf{R}_{i}$ é determinada sem invocar a TI, visto que, considerando tal hipótese, $E\left[\mathbf{x}(n) \mathbf{x}^{\mathrm{T}}(n-i)\right]=0$ para $i \neq 0$. Então, definindo

$$
\mathbf{K}_{i}(n)=E\left[\mathbf{v}(n) \mathbf{v}^{\mathrm{T}}(n-i)\right]
$$

(19) pode ser reescrita como

$$
E[e(n) e(n-i)]=E\left\{\operatorname{tr}\left[\mathbf{R}_{i} \mathbf{K}_{i}(n)\right]\right\} .
$$

Então, substituindo (21) em (12), obtém-se

$$
E[p(n)]=\beta E[p(n-1)]+(1-\beta) \sum_{i=1}^{N}\left\{\operatorname{tr}\left[\mathbf{R}_{i} \mathbf{K}_{i}(n)\right]\right\}^{2}
$$

Agora, para se obter o modelo desejado, é necessário estimar $\mathbf{R}_{i}$ e obter uma expressão para $\mathbf{K}_{i}(n)$. Assim, substituindo (13) em (3), a expressão de atualização do vetor de erro nos coeficientes pode ser escrita como

$$
\mathbf{v}(n+1)=\mathbf{v}(n)+\mu(n) e(n) \mathbf{x}(n) .
$$

Então, representando o sinal de erro como uma função de $\mathbf{v}(n)$, obtém-se

$$
\begin{aligned}
e(n) & =d(n)-\left[\mathbf{x}^{\mathrm{T}}(n) \mathbf{w}(n)\right] \\
& =d(n)-\mathbf{x}^{\mathrm{T}}(n) \mathbf{v}(n)-\mathbf{x}^{\mathrm{T}}(n) \mathbf{w}_{\mathrm{o}} .
\end{aligned}
$$

Agora, substituindo (24) em (23), tem-se

$$
\begin{aligned}
\mathbf{v}(n+1)= & \mathbf{v}(n)+\mu(n)[d(n) \mathbf{x}(n) \\
& \left.-\mathbf{x}(n) \mathbf{x}^{\mathrm{T}}(n) \mathbf{v}(n)-\mathbf{x}(n) \mathbf{x}^{\mathrm{T}}(n) \mathbf{w}_{\mathrm{o}}\right] .
\end{aligned}
$$

Dessa forma, como

$$
d(n)-\mathbf{x}^{\mathrm{T}}(n) \mathbf{w}_{\mathrm{o}}=\eta(n)
$$

pode-se escrever

$$
\mathbf{v}(n+1)=\mathbf{v}(n)+\mu(n)\left[\eta(n) \mathbf{x}(n)-\mathbf{x}(n) \mathbf{x}^{\mathrm{T}}(n) \mathbf{v}(n)\right]
$$

e

$$
\begin{gathered}
\mathbf{v}^{\mathrm{T}}(n+1-i)=\mathbf{v}^{\mathrm{T}}(n-i)+\mu(n-i)\left[\eta(n-i) \mathbf{x}^{\mathrm{T}}(n-i)\right. \\
\left.-\mathbf{v}^{\mathrm{T}}(n-i) \mathbf{x}(n-i) \mathbf{x}^{\mathrm{T}}(n-i)\right] .
\end{gathered}
$$

Fazendo, então, o produto $\mathbf{v}(n+1) \mathbf{v}^{\mathrm{T}}(n+1-i)$, tem-se

$$
\begin{aligned}
& \mathbf{v}(n+1) \mathbf{v}^{\mathrm{T}}(n+1-i)= \\
& \mathbf{v}(n) \mathbf{v}^{\mathrm{T}}(n-i) \\
& +\mu(n-i) \eta(n-i) \mathbf{v}(n) \mathbf{x}^{\mathrm{T}}(n-i) \\
& -\mu(n-i) \mathbf{v}(n) \mathbf{v}^{\mathrm{T}}(n-i) \mathbf{x}(n-i) \mathbf{x}^{\mathrm{T}}(n-i) \\
& +\mu(n) \eta(n) \mathbf{x}(n) \mathbf{v}^{\mathrm{T}}(n-i) \\
& -\mu(n) \mathbf{x}(n) \mathbf{x}^{\mathrm{T}}(n) \mathbf{v}(n) \mathbf{v}^{\mathrm{T}}(n-i) \\
& +\mu(n) \mu(n-i) \eta(n) \eta(n-i) \mathbf{x}(n) \mathbf{x}^{\mathrm{T}}(n-i) \\
& -\mu(n) \mu(n-i) \eta(n) \mathbf{x}(n-i) \mathbf{x}^{\mathrm{T}}(n-i) \mathbf{x}(n) \mathbf{v}^{\mathrm{T}}(n-i) \\
& -\mu(n) \mu(n-i) \eta(n-i) \mathbf{x}(n) \mathbf{x}^{\mathrm{T}}(n) \mathbf{v}(n) \mathbf{x}^{\mathrm{T}}(n-i) \\
& +\mu(n) \mu(n-i) \mathbf{x}(n) \mathbf{x}^{\mathrm{T}}(n) \mathbf{v}(n) \mathbf{v}^{\mathrm{T}}(n-i) \mathbf{x}(n-i) \mathbf{x}^{\mathrm{T}}(n-i) .
\end{aligned}
$$

Agora, tomando o valor esperado de (29), obtém-se

$$
\begin{aligned}
\mathbf{K}_{i}(n+1)= & \mathbf{K}_{i}(n)-E[\mu(n)] E\left[\mathbf{x}(n) \mathbf{x}^{\mathrm{T}}(n) \mathbf{v}(n) \mathbf{v}^{\mathrm{T}}(n-i)\right] \\
& -E \mu(n-i) E\left[\mathbf{v}(n) \mathbf{v}^{\mathrm{T}}(n-i) \mathbf{x}(n-i) \mathbf{x}^{\mathrm{T}}(n-i)\right] \\
& +E[\mu(n) \mu(n-i)]\left[\mathbf{x}(n) \mathbf{x}^{\mathrm{T}}(n) \mathbf{v}(n) \mathbf{v}^{\mathrm{T}}(n-i)\right. \\
& \left.\times \mathbf{x}(n-i) \mathbf{x}^{\mathrm{T}}(n-i)\right] .
\end{aligned}
$$

Finalmente, desenvolvendo (30) sob a luz da hipótese (iii) e utilizando o teorema de fatoração de momentos para sinais gaussianos [2], tem-se

$$
\begin{aligned}
\mathbf{K}_{i}(n+1)= & \mathbf{K}_{i}(n)-E[\mu(n)] \mathbf{R} \mathbf{K}_{i}(n)-E \mu(n-i) \mathbf{K}_{i}(n) \mathbf{R} \\
& +E[\mu(n)] E[\mu(n-i)]\left\{\mathbf{R}_{i} \operatorname{tr}\left[\mathbf{R}_{i} \mathbf{K}_{i}(n)\right]\right. \\
& +\mathbf{R} \mathbf{K}_{i}(n) \mathbf{R}+\mathbf{R}_{i} \mathbf{K}_{i}(n) \mathbf{R}_{i} .
\end{aligned}
$$

\section{B. Curva de Aprendizagem}

Utilizando (27), pode-se determinar uma expressão recursiva que descreva a evolução de $\mathbf{v}(n) \mathbf{v}^{\mathrm{T}}(n)$. Então,

$$
\begin{aligned}
\mathbf{v}(n+1) \mathbf{v}^{\mathrm{T}} & (n+1)=\mathbf{v}(n) \mathbf{v}^{\mathrm{T}}(n) \\
& -\mu(n) \mathbf{v}(n) \mathbf{v}^{\mathrm{T}}(n) \mathbf{x}(n) \mathbf{x}^{\mathrm{T}}(n) \\
& +\mu(n) \eta(n) \mathbf{v}(n) \mathbf{x}^{\mathrm{T}}(n) \\
& -\mu(n) \mathbf{x}(n) \mathbf{x}^{\mathrm{T}}(n) \mathbf{v}(n) \mathbf{v}^{\mathrm{T}}(n) \\
& +\mu^{2}(n) \mathbf{x}(n) \mathbf{x}^{\mathrm{T}}(n) \mathbf{v}(n) \mathbf{v}^{\mathrm{T}}(n) \mathbf{x}(n) \mathbf{x}^{\mathrm{T}}(n) \\
& -\mu^{2}(n) \eta(n) \mathbf{x}(n) \mathbf{x}^{\mathrm{T}}(n) \mathbf{v}(n) \mathbf{x}^{\mathrm{T}}(n) \\
& +\mu(n) \eta(n) \mathbf{x}(n) \mathbf{v}^{\mathrm{T}}(n) \\
& -\mu^{2}(n) \eta(n) \mathbf{x}(n) \mathbf{v}^{\mathrm{T}}(n) \mathbf{x}(n) \mathbf{x}^{\mathrm{T}}(n) \\
& +\mu^{2}(n) \eta^{2}(n) \mathbf{x}(n) \mathbf{x}^{\mathrm{T}}(n) .
\end{aligned}
$$

Agora, definindo $\mathbf{K}(n)=\mathrm{E}\left[\mathbf{v}(n) \mathbf{v}^{\mathrm{T}}(n)\right]$, tomando o valor esperado de (32), e considerando (i) e (ii), obtém-se

$$
\begin{gathered}
\mathbf{K}(n+1)=\mathbf{K}(n)-\mathrm{E}[\mu(n)][\mathbf{R K}(n)+\mathbf{K}(n) \mathbf{R}] \\
+\mathrm{E}^{2}[\mu(n)]\{\mathbf{R} \operatorname{tr}[\mathbf{R K}(n)]+2 \mathbf{R K}(n) \mathbf{R}\} \\
+\mathrm{E}^{2}[\mu(n)] \mathbf{R} \sigma_{\eta}^{2} .
\end{gathered}
$$

Por fim, a expressão que determina a evolução da curva de aprendizagem é obtida de

$$
J(n)=\mathrm{E}\left[e^{2}(n)\right]=\sigma_{\eta}^{2}+\operatorname{tr}[\mathbf{R K}(n)] .
$$

\section{Resultados de Simulação}

Nesta seção, são apresentados resultados de simulação numérica visando verificar tanto o desempenho do algoritmo (apresentado em [14]) com complexidade computacional reduzida (Tabela II) quanto a precisão do modelo 
desenvolvido. Para tal, considera-se um problema de identificação de sistema, utilizando o mesmo cenário descrito em [13] e [14]. A planta desconhecida é dada pelo vetor $\mathbf{w}_{\mathrm{o}}=\left[\begin{array}{llll}5 & 0 & 1 & 8\end{array}\right]^{\mathrm{T}}$. O sinal de entrada é correlacionado, gaussiano, com média zero e variância $\sigma_{x}^{2}=1,56$, obtido de um processo $\mathrm{AR}(1)$, dado por $x(n)=a x(n-1)+u(n)$, com $a=0,6$ e $u(n)$ sendo um ruído branco gaussiano com média zero e variância $\sigma_{u}^{2}=1$. A dispersão de autovalores da matriz $\mathbf{R}$ é $\chi=8,5$. Além disso, um ruído aditivo branco gaussiano $\eta(n)$ com média zero e variância $\sigma_{\eta}^{2}=1$ é adicionado à saída da planta. Por razões de estabilidade do algoritmo, o valor máximo do passo de adaptação variável $\mu(n)$ é limitado em 0,01 .

\section{A. Exemplo 1}

Neste exemplo, são mostrados resultados de simulação visando comparar o desempenho do algoritmo apresentado em [14], nas suas duas versões (padrão e simplificada) (ver Tabela II), com os algoritmos de Kwong [10] e Aboulnasr [13]. Os resultados são obtidos por simulação Monte Carlo (500 rodadas independentes) e estão mostrados nas Figs. 2 e 3. Os parâmetros para todos os algoritmos são selecionados de forma a se obter o mesmo erro quadrático médio (EQM) em excesso (final) de $-30 \mathrm{~dB}$. A Fig. 2 ilustra a evolução do EQM em excesso, definido por $E\left\{[e(n)-\eta(n)]^{2}\right\}$, para os quatro algoritmos considerados. A Fig. 3 mostra (também para os quatro algoritmos) a evolução do componente $w_{1}(n)$ do vetor de coeficientes. Observa-se dessas figuras que ambas as versões do algoritmo proposto em [14] (padrão e simplificado) apresentam um desempenho superior ao dos outros algoritmos avaliados ([10] e [13]) para o cenário de simulação considerado. Verifica-se ainda que o algoritmo com complexidade reduzida (versão simplificada do algoritmo proposto em [14]) não apresenta qualquer perda de desempenho em relação ao algoritmo [14] padrão.

\section{B. Exemplo 2}

Neste exemplo, resultados de simulação obtidos pelo método Monte Carlo (MC) e através do modelo proposto são comparados. As Figs. 4, 5 e 6 mostram tais resultados. A Fig. 4 ilustra a evolução do passo de adaptação variável, a Fig. 5, o comportamento médio do vetor de coeficientes, e a Fig. 6, a curva de aprendizagem (EQM). A partir dessas figuras, constata-se o bom casamento entre os resultados de simulação $\mathrm{MC}$ e as correspondentes predições obtidas com o modelo proposto. As pequenas discrepâncias, observadas na fase transitória das curvas, são devidas às aproximações utilizadas no desenvolvimento do modelo.

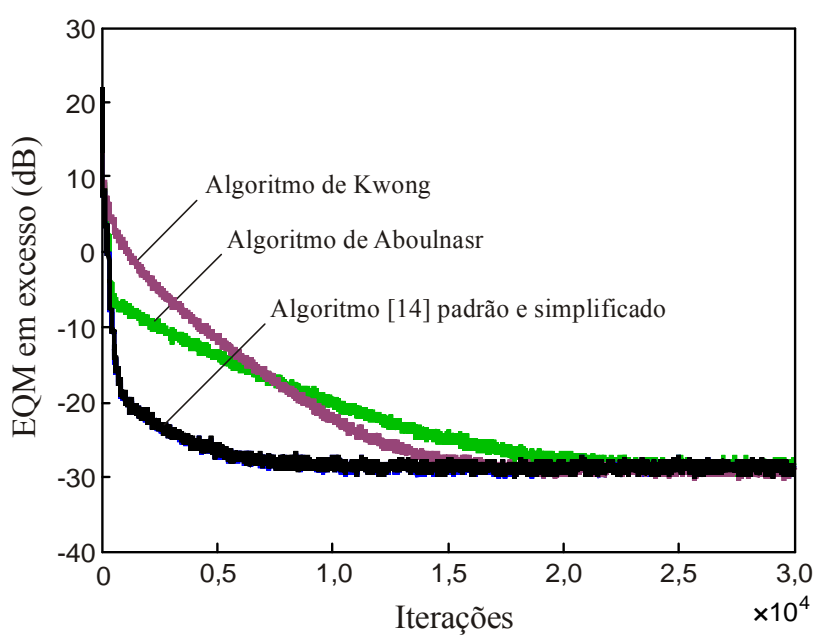

Fig. 2. Curvas do EQM em excesso para os algoritmos avaliados.

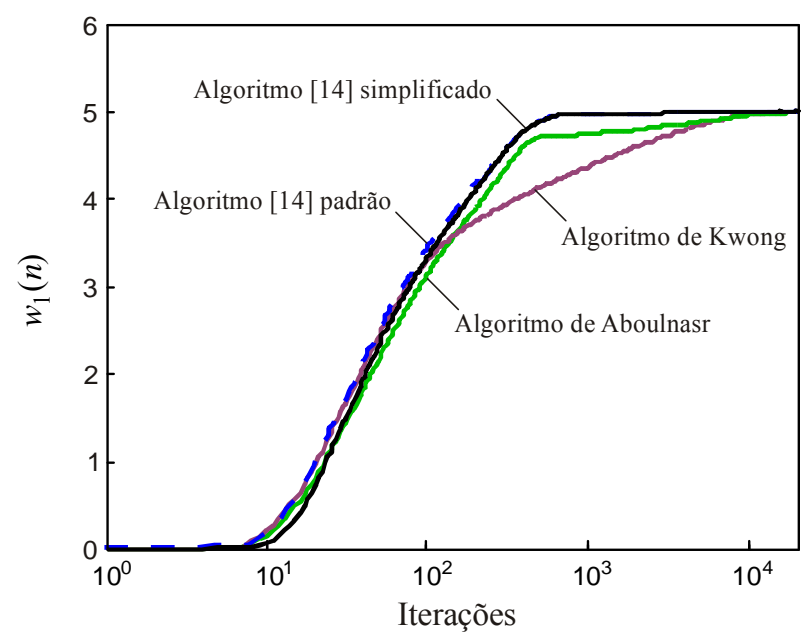

Fig. 3. Evolução do coeficiente $w_{1}(n)$ para os algoritmos avaliados.

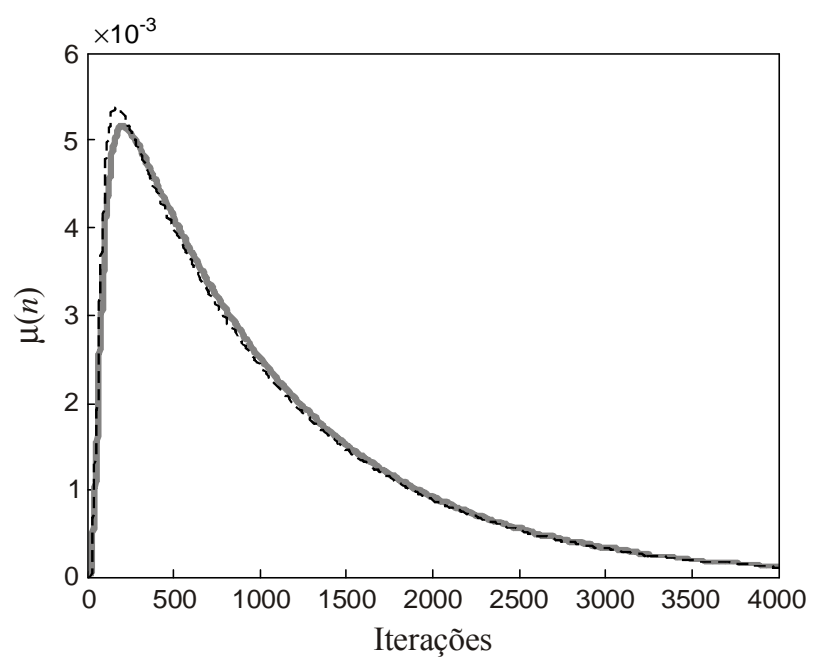

Fig. 4. Evolução do passo variável de adaptação. (Linha cinza) simulação. (Linha tracejada escura) modelo proposto (10). 


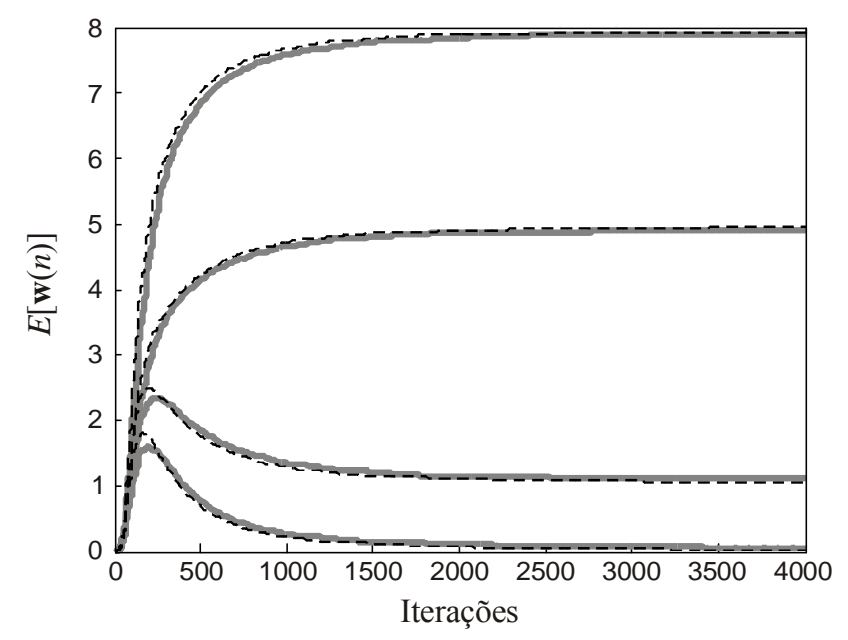

Fig. 5. Comportamento médio do vetor de coeficientes. (Linhas cinza) simulação MC. (Linhas tracejadas escuras) modelo proposto (9).

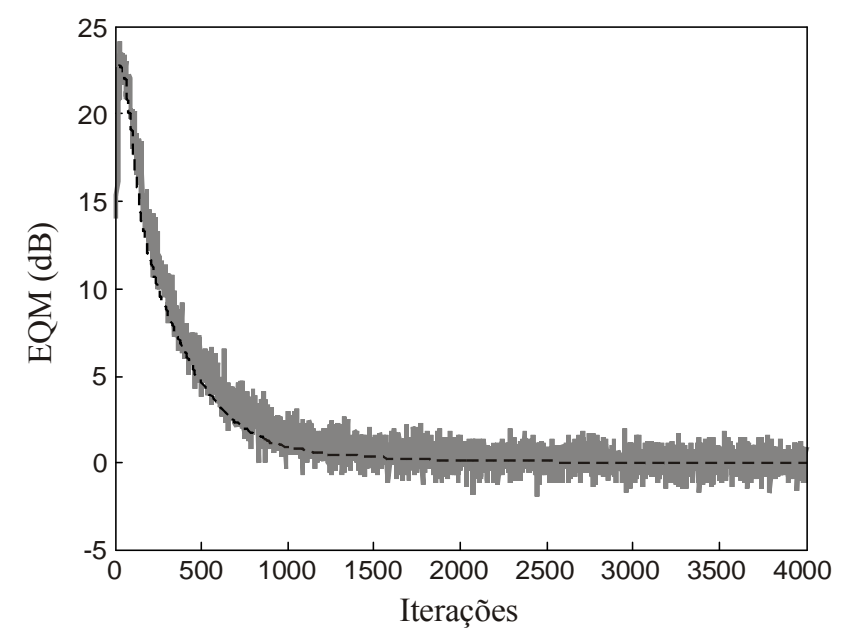

Fig. 6. Curva de aprendizagem (EQM). (Linha irregular cinza) simulação MC. (Linha tracejada escura) modelo proposto (34).

\section{CONCLUSÕES}

Neste trabalho, um modelo estocástico do algoritmo LMS de passo variável apresentado em [14], para ambientes com baixa razão sinal-ruído, é proposto. Tal algoritmo considera a autocorrelação do sinal de erro usando N lags. Adicionalmente, é também discutida uma proposta de redução da carga computacional do supramencionado algoritmo. Resultados de simulações numéricas confirmam a boa precisão do modelo desenvolvido.

\section{REFERÊNCIAS}

[1] B. Widrow and M. Hoff, "Adaptive switching circuits," in Proc. IRE Western Electronic Show and Convention, New York, USA, Part 4, Aug. 1960, pp. 96-104.

[2] S. Haykin, Adaptive Filter Theory, $4^{\text {th }}$ ed. Upper Saddle River, NJ: Prentice Hall, 2002.

[3] J. C. Richards, M. A. Webster, and J. C. Principe, "A gradient-based variable step-size LMS algorithm," in Proc. IEEE Southeastcon, Williamsburg, USA, vol. 2, Apr. 1991, pp. 1083-1087.
[4] V. J. Mathews and Z. Xie, "A stochastic gradient adaptive filter with gradient adaptive step size," IEEE Trans. Signal Process., vol. 41, no. 6, pp. 2075-2087, June 1993.

[5] A. I. Sulyman and A. Zerguine, "Convergence and steady state analysis of a variable step-size normalized LMS algorithm," in Proc. IEEE Int. Symp. Signal Processing and Its Applications (ISSPA), Paris, France, vol. 2, July 2003, pp. 591-594.

[6] B. Farhang-Boroujeny, "Variable step size LMS algorithm - New developments and experiments," IEE Proceedings - Vision, Image, Signal Process., vol. 141, no. 5, pp. 311-317, Oct. 1994.

[7] T. J. Shan and T. Kailath, "Adaptive algorithms with an automatic gain control feature," IEEE Trans. Circuits Syst., vol. CAS-35, no. 1, pp. 122-127, Jan. 1988.

[8] J. Okello, Y. Itoh, Y. Fukui, I. Nakanishi, and M. Kobayashi, "A new modified variable step size for the LMS algorithm," in Proc. IEEE Int. Symp. Circuits and Systems (ISCAS), Monterey, USA, vol. 5, Jun. 1998, pp. 170-173.

[9] W. P. Ang and B. Farhang-Boroujeny, "A new class of gradient adaptive step-size LMS algorithms," IEEE Trans. Signal Process., vol. 49, no. 4, pp. 805-810, Apr. 2001.

[10] R. H. Kwong and E. W. Johnston, "A variable step size LMS algorithm," IEEE Trans. Signal Process., vol. 40, no. 7, pp. 1633-1642, July 1992.

[11] I. Nakanishi and Y. Fukui, "A new adaptive convergence factor algorithm with the constant damping parameter," IEICE Trans. Fundamentals, vol. E78-A, no. 6, pp. 649-655, Jun. 1995.

[12] M. H. Costa and J. C. M. Bermudez, "A robust variable step size algorithm for LMS adaptive filters," in Proc. IEEE Int. Conf. Acoust., Speech, Signal Process., Toulouse, France, vol. 3, May 2006, pp. 93-96.

[13] T. Aboulnasr and K. Mayyas, "A robust variable step-size LMS-type algorithm: analysis and simulations," IEEE Trans. Signal Process., vol. 45, no. 3, pp. 631-639, Mar. 1997.

[14] J. G. F. Zipf, O. J. Tobias, R. Seara, "A new VSSLMS algorithm based on error autocorrelation," in Europ. Signal Process. Conf. (EUSIPCO), Lausanne, Switzerland, Aug. 2008, pp. 1-5.

[15] D. W. Kim, J. H. Hoi, Y. S. Choi, C. H. Jeon, and H. Y. Ko, "A VS-LMS algorithm using normalized absolute estimation error," in Proc. IEEE Digital Signal Processing Applications (TENCON), Perth, Australia, vol. 2, Nov. 1996, pp. 692-697.

[16] Z. Ramadan and A. Poularikas, "A robust variable step-size LMS algorithm using error-data normalization," in Proc. IEEE Southeastcon, Huntsville, USA, Apr. 2005, pp. 219-224.

[17] B. Rohani and K. S. Chung, "A modified LMS algorithm with improved convergence," in Proc. IEEE Singapore Int. Conf. Communication Systems, Singapore, Nov. 1994, pp. 845-849.

[18] D. L. Duttweiler, "Proportionate normalized LMS adaptation in echo cancellers," IEEE Trans. Speech Audio Process., vol. 8, no. 5, pp. 508-518, Sept. 2000.

[19] J. Benesty and S. L. Gay, "An improved PNLMS algorithm," in Proc. IEEE Int. Conf. Acoust., Speech, Signal Process. (ICASSP), Orlando, USA, May 2002, pp. 1881-1884.

[20] Y. Wei and S. B. Gelfand, "Noise-constrained least mean squares algorithm," IEEE Trans. Signal Process., vol. 49, no. 9, pp. 1961-1970, Sep. 2001. 